\title{
EXISTENCE AND UNIQUENESS OF INTEGRAL FUNCTION APPROXIMATIONS
}

\author{
by \\ Allan W. McInnes \\ Department of Mathematics, University of Canterbury, Christchurch, N.Z.
}

No.52

October, 1989.

\begin{abstract}
The problem of approximating a real-valued, locally analytic function by an integral function is considered. Results are obtained for the existence, uniqueness and order of approximation for both 'normal' and 'non-normal' cases. Some illustrative examples are given.
\end{abstract}

Subject classification: AMS(MOS) : 41A21; CR : G.1.2.

Key words and phrases: integral function approximation, order of approximation, Hermite-Padé approximation. 


\section{\$1. Introduction.}

This paper considers the problem of approximating a real-valued, locally analytic function by an integral function, where the approximations are determined by sufficient derivative information about the given function at the origin. The objective is to investigate these approximations in the same way that approximations by algebraic functions were previously studied $[7,8]$. The main result is that analogous results for the order of approximation are obtained.

The class of integral functions of degree $p$ - defined formally in the next section - consists of those functions which are the solution of a linear homogeneous ordinary differential equation of order $p$ with polynomial coefficients. This name has been chosen for the class analogous to algebraic functions following Stahl [9], since the solutions to differential equations are classically called integral curves. Thus we introduce an approximating subset which is much larger than usually contemplated. The class includes polynomials, algebraic functions and the usual elementary functions. However, apart from equations with constant coefficients, the general linear equation of order $p$ cannot be explicitly integrated in terms of elementary functions. In general, the equations which arise from problems in applied mathematics and which cannot be reduced to equations with constant coefficients, have solutions which are transcendental functions. The class of integral functions includes the well-known transcendental functions like the Bessel functions, but does not include functions like the Riemann-Zeta function which does not satisfy an ordinary differential equation of finite order. As would reasonably be expected, functions which satisfy a linear homogeneous differential equation, such as the Legendre, Chebyshev and Bessel functions, are reproduced by this approximation.

As noted by Baker and Lubinsky [2] and Baker and Graves-Morris [1] and the references therein, these approximants have already been applied to various calculations in mathematical physics. A variety of generalizations of the Taylor polynomial approximation have been suggested and are often collected in the class of what are called Hermite-Pade approximants [2,9]. Della Dora and Di Crescenzo [5] developed the theory in a general framework and noted explicitly that their formulation included as particular cases what we have called approximation by algebraic functions [7] and approximation by integral functions. Stahl [9] considers the asymptotics of 'integral Hermite-Padé approximants', and Baker and Lubinsky [2] investigated the convergence of 'differential Hermite-Padé approximations'.

The basic definitions used in this paper are similar to those used by Baker and Lubinsky [2], although these authors use the inhomogeneous form of the differential equation. In this paper we restrict attention to the approximation of a locally analytic function and consider local 
approximation, whereas Baker and Lubinsky considered the more general problem when the given function is meromorphic. On the other hand the Baker and Lubinsky formulation for rows, for an analytic function reduces to the problem of considering polynomial approximations to the function.

Following the approach in [7], a careful distinction is made between the approximation of the integral form which determines the polynomial coefficients of the differential equation for the integral function, and the approximating properties of the integral function itself. The distinction between these two concepts leads to the separation of degenerate cases arising from different sources, and consequently a clear treatment of the effects of each type. As in [7], this leads to the definition of the surplus in the case of the integral form, and the definition of deficiency in the case of approximation by the integral function.

In Section 2 the problem of the approximation of the integral form which determines the coefficient polynomials is considered. The concept of the surplus in the approximation of the integral form is introduced. In Section 3 the approximating properties of the integral function are investigated. The concept of the deficiency of the approximation is introduced and the order of approximation by an integral function is quantified. Section 4 contains a series of illustrative examples to demonstrate the results of the previous sections in particular cases.

\section{\$2. The Integral Form.}

Consider the problem of approximating a real-valued, locally analytic function $f(x)$, by an integral function, $Q(x)$. We may suppose that the function $f(x)$ is analytic in the neighbourhood of the origin.

\section{DEFINITION 1 (INTEGRAL FUNCTION).}

Let $\mathbf{n}=\left(n_{0}, n_{1}, \cdots, n_{p}\right)$, where $n_{i} \geq-1$ are integers for $i=0(1) p$, and $p \geq 1$.

An $\mathbf{n}$ integral function of degree $p$ is a function $Q(x)$ which satisfies

$$
P_{\mathbf{n}, p}(Q, x) \equiv \sum_{i=0}^{p} a_{i}(x) Q^{(i)}(x)=0
$$

where the $a_{i}(x)$ are algebraic polynomials with degree $\left(a_{i}(x)\right) \leq n_{i}$ for $i=0(1) p$, and $Q^{(i)}(x)$ is the $i$ th formal derivative of $Q(x)$.

By convention, the polynomial of degree -1 is identically zero. Generally the subscripts $\mathbf{n}$, $p$ on $P$ will be dropped when the context makes them obvious. 
The integral function $Q(x)$ is a solution of the linear, homogeneous $p$ th order differential equation (1). It is well-known that in general the solutions to the differential equation (1) form a linear space of dimension $p$. A unique solution may be determined by given initial conditions which will be imposed later. Note that the integral function will be regarded as being defined by the polynomial coefficients $a_{i}(x)$, in an analogous way that the algebraic function is also regarded as being defined by its polynomial coefficients [7].

DEFINITION 2 (INTEGRAL FORM).

Let $f(x)$ be a formal power series. Let $p \geq 1$ and $\mathbf{n}=\left(n_{0}, n_{1}, \cdots, n_{p}\right)$ where $n_{i} \geq-1$ are integers for $i=0(1) p$. The function

$$
P_{\mathbf{n}, p}(f, x) \equiv \sum_{i=0}^{p} a_{i}(x) f^{(i)}(x)=O\left(x^{N}\right)
$$

will be called an $\mathbf{n}$ integral form of degree $p$, where the $a_{i}(x)$ are algebraic polynomials with degree $\left(a_{i}(x)\right) \leq n_{i}$ for $i=0(1) p, N+1=\sum_{i=0}^{p}\left(n_{i}+1\right)$, and $f^{(i)}(x)$ is the $i$ th formal derivative of $f(x)$.

Note that $P(f, x)$ may also be written as a formal power series, $r(x)=\sum_{i=0}^{\infty} r_{i} x^{i}$, and that the conditions (2) are equivalent to the requirement that the linear functionals

$$
D^{k}(P(f, x))=D^{k} r(x)=r^{(k)}(0) / k !=0 \quad \text { for } k=0(1) N-1
$$

If $p=2$, the problem reduces to a general form of the $G^{3} J$ approximants [1], and if $p=1$ the problem reduces to Baker's D-log approximant [1].

The existence of the polynomial coefficients $a_{i}(x)$ in $(2)$ is essentially given in [2], but the theorem is included here for completeness.

\section{THEOREM 3 (EXISTENCE).}

There always exists an $\mathbf{n}$ integral form of degree $p$ for a given formal power series $f(x)$. This integral form may be assumed to satisfy

$$
\overline{\mathbf{a}}(x)=\left(a_{1}(x), \cdots, a_{p}(x)\right) \not \equiv 0
$$


PROOF:

The integral form is defined by the coefficient polynomials $a_{i}(x)$. The existence of the $a_{i}(x)$ follows since the application of the linear functionals $D^{k}, k=0(1) N-1$, (where $\left.D^{k} r(x)=r^{(k)}(0) / k !\right)$ to (2) leads to a system of $N$ homogeneous linear equations for the $N+1$ unknown coefficients of the $a_{i}(x)$. Hence a non-trivial solution, with the $a_{i}(x)$ not all zero, exists.

If the $a_{i}(x), i=1(1) p$, are chosen to be identically zero then (2) becomes a trivial equation. If the $a_{i}(x) \equiv 0$ for $i=1(1) p$, then provided $f(0) \neq 0$ we have $a_{0}(x) \equiv 0$. We may always consider $g(x)$ if $f(x)=x^{r} g(x), r>0, g(0) \neq 0$, and hence for a non-trivial solution to a non-trivial equation we require $\overline{\mathbf{a}}(x)=\left(a_{1}(x), \cdots, a_{p}(x)\right) \not \equiv 0$.

As in the case of algebraic functions [7], the uniqueness of this integral form requires a more careful argument. The matrix form of the system of linear equations represented by (2) has the coefficient matrix

$$
F=\left[F_{n_{0}}: F_{n_{1}}: \cdots: F_{n_{p}}\right]
$$

where

$$
F_{n_{k}}=\left[\begin{array}{cccc}
d_{k} f_{k} & & & \\
d_{k+1} f_{k+1} & d_{k} f_{k} & & \\
\cdot & \cdot & & \\
d_{k+n_{k}} f_{k+n_{k}} & d_{k+n_{k}-1} f_{k+n_{k}-1} & \cdots & d_{k} f_{k} \\
\cdot & \cdot & & \cdot \\
d_{k+N-1} f_{N+k-1} & d_{k+N-2} f_{k+N-2} & \cdots & d_{k+N-1-n_{k}} f_{k+N-1-n_{k}}
\end{array}\right]
$$

where

$f(x)=\sum_{i=0}^{\infty} f_{i} x^{i}$, and the constants $d_{j}=j ! /(j-k) !$ with the convention that $0 !=1$.

The matrix $F$ has dimension $N \times(N+1)$ and hence has a solution space of dimension at least 1. If the rank of the matrix $F$ is $N$ (and hence the solution space has dimension exactly $1)$, then any constant multiple of the coefficient polynomials $\left\{a_{i}(x)\right\}_{i=0}^{p}$ will also be a solution. These solutions may be called essentially unique [2]. A unique representative of this class of solutions may be defined by choosing any suitable normalization of the non-trivial coefficients. Thus if $\overline{\mathbf{a}}$ represents the vector of coefficients of the non-trivial vector $\overline{\mathbf{a}}(\mathrm{x})$, then $\|\overline{\mathbf{a}}\|=1$ for some convenient norm may be used. The use of the $l_{\infty}$ norm on this vector is in fact the normalization suggested by Baker and Lubinsky [2]. However, the implied normalization of $a_{2}^{\prime}(0)=1$ used in [1] for the $G^{3} J$ approximants (a particular form of the case $p=2$ ) is not 
a suitable normalization in general since there is no requirement that $a_{2}^{\prime}(0) \neq 0$ in the general case for $p=2$.

The situation is less clear when the solution spacxe is multi-dimensional. This may occur when the matrix has rank $<N$. If the rank of $F$ is $N+1-k$, then the solution space of the integral form has dimension $k>1$. This situation for the case of algebraic forms was discussed in [7,8].

\section{DEFINITION 4 (ORDER OF THE INTEGRAL FORM).}

The order of the $\mathbf{n}$ integral form is defined to be

$$
R=\operatorname{Ord}(P(f, x)) \text { if } P(f, x)=O\left(x^{R}\right), \neq O\left(x^{R+1}\right) \text { as } x \rightarrow 0
$$

If the solution space of equation (2) is multi-dimensional, then the order of the integral form is to be interpreted as the minimum of the orders of the multiple solutions.

Note that if $P(f, x)=0$ in Definition 4 , then $f(x)$ is in fact an integral function and we may regard the order $R=\infty$ in this case. It may be observed that if $P_{\mathbf{n}, p}(f, x)=0$ then for any integer $k, D^{k} P_{\mathbf{n}, p}(f, x)=0$, which may also be expressed as $P_{\mathbf{m}, p+k}(f, x)=0$ for a suitable $\mathbf{m}$ (with $m_{p+k}=n_{p}$ ). Hence if $f(x)$ is an integral function, the most appropriate integral form $P_{\mathbf{n}, p}(f, x)$ may be the minimum value of $p$ for which this form vanishes. But in general, for a fixed degree $p$, we seek the order $R$ as large as possible since this will give a better approximation.

If the dimension of the solution space is $k>1$, then to obtain a unique representative we seek a one-dimensional subspace whose elements satisfy $P(f, x)=O\left(x^{R}\right)$ where the order $R$ is maximal over the space of $\mathbf{n}$ integral forms of degree $p$. For example if $P(f, x)$ has order $R$ and $\mathbf{a}^{(i)}(x)=\left(a_{o}^{(i)}(x), \ldots, a_{p}^{(i)}(x)\right), i=1,2$, are two linearly independent solutions to $(2)$, then by taking a suitable linear combination, $c_{1} \mathrm{a}^{(1)}(x)+c_{2} \mathrm{a}^{(2)}(x)$, of these solutions, the term $\left(c_{1} r_{R}^{(1)}+c_{2} r_{R}^{(2)}\right) x^{R}$ may be eliminated and an $\mathbf{n}$ integral form of order at least $R+1$ is obtained, with the dimension of the solution space decreased by 1 .

\section{THEOREM 5 (UNIQUENESS).}

There always exists an essentially unique $\mathbf{n}$ integral form of degree $p$, which is of maximal order $R \geq N$, and which may be chosen uniquely by a suitable normalization of the coefficients of the coefficient polynomials. This unique representative will be denoted by $P^{*}(f, x)$. 


\section{PROOF:}

If the coefficient matrix $F$ (equation (3)), has rank $N$ then the solution space has dimension 1 and the result is trivial.

If the matrix $F$ has rank $N+1-k$ for $k>1$, then the solution space has dimension $k$. Suppose $P(f, x)$ has order $R$ such that $N \leq R$, and let $\mathbf{a}^{(i)}(x), i=1(1) k$ represent a basis for this solution space.

Then $\sum_{i=1}^{k} c_{i} \mathbf{a}^{(i)}(x)$ represents an $\mathbf{n}$ integral form of order at least $R+k-1$, where the constants $c_{i}$ are defined by the linear system

$$
\sum_{i=1}^{k} c_{i} r_{R+j}^{(i)}=0, \quad j=0(1) k-2,
$$

and where the $r_{R+j}$ are the coefficients of $x^{R+j}$ of the formal power series associated with $P(f, x)=r(x)$, i.e. $P(f, x)=r(x)=\sum_{i=0}^{\infty} r_{i} x^{i}$.

If the matrix of the linear system (4) has rank $k-1$, then there exists an essentially unique solution. However, if this matrix has rank $<k-1$, then we must iterate this process since there are still linearly independent solutions. Since the rank reduces by at least one at each step, the number of iterations must be finite, noting of course, that if we obtain a zero matrix or a zero value for the integral form then $R=\infty$ and the exact integral form, and hence the exact integral function, has been obtained.

A unique representative of this essentially unique solution lying in a one-dimensional space, may be obtained by a suitable normalization of the coefficients. That is if $\overline{\mathbf{a}}$ represents the vector of coefficients of the non-trivial vector $\overline{\mathbf{a}}(x)$, then $\|\overline{\mathbf{a}}\|=1$ for some convenient norm may be used.

An example of the situation when the process in the proof of Theorem 5 is necessary was given in [7] for the case of the algebraic form. It was noted that if $f(x)$ is an even function, the $n_{k}$ are all even and $p=2,4$, then an examination of the coefficient matrix $F$ reveals that the matrix has rank of at most $N-1$, and hence the solution space has dimension of at least 2 .

In fact the situation is more general and applies to both algebraic and integral forms since the basic structure of the coefficient matrix $F$ is similar under certain circumstances. If $f(x)$ is an even function then odd derivatives of $f(x)$ are odd, but even derivatives of $\mathrm{f}(\mathrm{x})$ are even. Hence by considering integral forms $P(f)$ in which the "basis" functions are even derivatives, the coefficient matrix $F$ for $f(x)$ an even function has a similar structure to that of the algebraic form for $f(x)$ an even function. 
THEOREM 6 (MULTIPLE SOLUTIONS).

If $f(x)$ is an even function, the $n_{k}$ are all even and the degree $p$ is even, then the coefficient matrix $F$, for the integral form with a "basis" of even derivatives (and hence $n_{k} \geq 0$ for all $k$ ), has rank at most $N-p / 2$ and hence the solution space has dimension at least $p / 2+1$.

\section{PROOF:}

Under the hypotheses of the theorem $N$ is even, and $N+1$ is odd..

The coefficient matrix $F$ has its $N+1$ columns partitioned in $p+1$ blocks as given by (3). Let the columns be rearranged into blocks of up to $p+1$ columns, in the following way. The first block consists of the first columns from each of the blocks $F_{n_{k}}, k=0(1) p$. The second block of up to $p+1$ columns consists of the third columns from each of the blocks $F_{n_{b}}, k=0(1) p$. If any block $F_{n_{k}}$ does not have a third column then this null column is ignored and the block in the rearranged matrix will have less than $p+1$ columns. Continuing in this manner, when all the odd numbered columns of the blocks $F_{n_{k}}$ have been dealt with, the procedure is repeated for the even numbered columns of the blocks $F_{n_{k}}$.

The rows of the resulting matrix are now interchanged so that the odd numbered rows are collected into the first $N / 2$ rows and the even numbered rows are collected into the final $N / 2$ rows.

Thus the matrix now has the form

$$
\left[\begin{array}{ll}
A & 0 \\
0 & B
\end{array}\right]
$$

where $A, B$ both have $N / 2$ rows.

If $n_{k}=0, k=0(1) p$, then the matrix $B$ in (5) is null, $N=p$, and $A$ has $p / 2$ rows of zeros. Hence the matrix (5) has rank at most $p / 2=N-p / 2$.

If $n_{k}>0$ for at least one value of $k$ then the corresponding block $F_{n_{k}}$ in (3) has one more odd numbered column than even numbered column, since $n_{k}$ is even and $F_{n_{k}}$ has $n_{k}+1$ columns. Hence $A$ has $p+1$ more columns than $B$. By considering a Laplacian expansion of the determinant of an arbitrary $N \times N$ submatrix of (5), in terms of the first $N / 2$ rows, it is clear that each cofactor has at least $p / 2$ columns of zeros. Hence the rank of (5) (and hence of $F$ ) is at most $N-p / 2$.

The rank of the matrix $F$ may in fact be even less than $N-p / 2$ if the ranks of $A$ or $B$ are not full. 


\section{\$2.1. Degeneracies in the Integral Form.}

In the integral form defined by equation (2) it is possible that some of the coefficient polynomials are not of full degree, i.e. $\operatorname{deg}\left(a_{i}(x)\right)<n_{i}$ for some $i$. Except when all the coefficient polynomials are not of full degree, this is of no particular consequence. It may be compared to the occurrence in the algebraic function case when the Taylor polynomial approximation of degree 1 to the function $f(x)=1+x^{2}$ is given by $p_{1}(x)=1$, i.e. there is a zero coefficient of the term in $x$.

Of greater importance is the fact that a particular set of coefficient polynomials which solves (2), may in fact eliminate more of the coefficients of $r(x)$ than just the first $N$. A simple example for $p=1$ is the $(0,0)$ approximation to $f(x)=1+(x-1)^{2}$. In this case $N=1$, but the integral form

$$
\text { 1. } f(x)+1 . f^{\prime}(x)=O\left(x^{2}\right) \text {. }
$$

In $[7,8]$ the term "surplus" was used to describe this phenomenon for approximation by algebraic functions.

\section{DEFINITION 7 (SURPLUS OF INTEGRAL FORM).}

The surplus, $S(\mathbf{n})$, of the $\mathbf{n}$ integral form $P^{*}(f, x)$ is defined by

$$
S(\mathbf{n})=\operatorname{Ord}\left(P^{*}(f, x)\right)-N
$$

where the order of the integral form is defined in Definition 4.

The surplus, $S(\mathbf{n})=S \geq 0$, is the amount of extra matching obtained from $P^{*}(f, x)$. This may be achieved by serendipity for a particular function (as in the simple example above), or by the process of obtaining a unique solution as outlined in the proof of Theorem 5 . It is clear that in general we would like the surplus to be as large as possible since if $S=\infty$ then the integral form represents an integral function exactly.

In $[7,8]$, the surplus was used to define an $S$-table of the algebraic forms. It was indicated that in the rational case $(p=1)$, this table illustrates the block structure of the Pade table in a somewhat easier fashion than the traditional $C$-table [1]. In the case of integral forms of degree $p$, the $S$-table of the integral forms would be a $(p+1)$ - dimensional table. The structure of this table could be expected to lead to the structure of the table for the $\mathbf{n}$ integral forms of degree $p$ in an analogous way. An analogous theorem for basic block structure can also be given for the integral forms. 
THEOREM 8 (BASIC BLOCK STRUCTURE).

If the $\mathbf{n}$ integral form of degree $p, P_{\mathbf{n}}^{*}(f, x)$, has surplus $S(\mathbf{n})=S>0$, then

$$
x^{r} P_{\mathbf{n}}^{*}(f, x), \quad r=0(1)(S / p)
$$

is an $\mathbf{m}$ integral form with a surplus of $S(\mathbf{m})=S(\mathbf{n})-p r-\sum_{k=0}^{p} i_{k}$, where

$$
\begin{gathered}
\mathbf{m}=\left(m_{0}, m_{1}, \ldots, m_{p}\right) \quad \text { with } \\
m_{k}=n_{k}+r+i_{k}, \quad i_{k} \geq 0 \text { for } k=0(1) p,
\end{gathered}
$$

satisfies

$$
\sum_{k=0}^{p} i_{k} \leq S-p r
$$

PROOF:

Since $\quad \operatorname{Ord}\left(P_{\mathbf{n}}^{*}(f, x)\right)=N+S, \quad$ then

$$
\operatorname{Ord}\left(x^{r} P_{\mathbf{1}}^{*}(f, x)\right)=N+S+r
$$

Hence $x^{r} P_{\mathbf{n}}^{*}(f, x)$ will be an $\mathbf{n}+$ ro (where $\mathbf{o}=(1,1, \cdots, 1)$ ) integral form of degree $p$ provided $p r \leq S$. The surplus of this integral form, $P_{\mathbf{n}+r o}(f, x)$, is $S-p r$. Further, this integral form is also an integral form of the type $\mathbf{m}$ where $\mathbf{m}=\left(m_{0}, m_{1}, \cdots, m_{p}\right)$ and $m_{k}=n_{k}+r+i_{k}, i_{k} \geq 0$, for $k=0(1) p$, satisfies $\sum_{k=0}^{p} i_{k} \leq S-p r$.

This follows since $P_{\mathbf{m}}(f, x)=O\left(x^{M+S(\mathbf{m})}\right)$ with $M+1=\sum_{k=0}^{p}\left(m_{k}+1\right)$. Hence $\operatorname{Ord}\left(P_{\mathbf{m}}(f, x)\right)=M+S(\mathbf{m})=N+S+r$ (from above). Substituting for $M$ and $N$, and using the relation between $m_{k}$ and $n_{k}$ gives

$$
\sum_{k=0}^{p} m_{k}+p+S(\mathbf{m})=\sum_{k=0}^{p} n_{k}+p+S+r .
$$

Hence $\sum_{k=0}^{p} i_{k}+p r+S(\mathbf{m})=S$ and $S(\mathbf{m}) \geq 0$ gives the required relation.

Further details on the block structure and the occurrence of overlapping blocks will be given in a future report. Some simple examples of the basic structure can be given. 


\section{EXAMPLE 9.}

Let $p=1$ and $f(x)=1+(x-1)^{2}$.

The $(0,0)$ integral form for this function has the polynomial coefficients $a_{0}(x)=1$, $a_{1}(x)=1$. The integral form is

$$
1 f(x)+1 \cdot f^{\prime}(x)=O\left(x^{2}\right)
$$

Since $N=1$ this integral form has surplus $S=1$. Hence it is also an integral form of types $(0,1)$ and $(1,0)$. The $(1,1)$ integral form has polynomial coefficients $x a_{0}(x)=x, x a_{1}(x)=x$, with surplus $S=0$.

EXAMPLE 10.

Let $p=1$ and $f(x)=\cos x$.

The $(1,0)$ integral form is

$$
x f(x)+f^{\prime}(x)=O\left(x^{3}\right)
$$

Since $N=2$, we have $S=1$, and this is also the integral form of types $(2,0),(1,1)$. The integral form of type $(2,1)$ is

$$
x^{2} f(x)+x f^{\prime}(x)=O\left(x^{4}\right)
$$

with $S=0$.

\section{2 §3. The Integral Approximation.}

Once the unique $\mathbf{n}$ integral form of degree $p, P^{*}(f, x)$, satisfying equation (2) has been obtained, it is clear that we can define an $\mathbf{n}$ integral function of degree $p, Q(x)$, which satisfies the equation

$$
P^{*}(Q, x) \equiv \sum_{i=0}^{p} a_{i}^{*}(x) Q^{(i)}(x)=0
$$

Since the coefficients, $a_{i}^{*}(x)$, are determined by the given function $f(x)$, this function, $Q(x)$, represents an approximation of $f(x)$. Recall that $f(x)$ is assumed given in the sense that a sufficient number of derivative values at the origin are known.

From the general theory of linear ordinary differential equations [6], it is known that equation (6) ordinarily has $p$ linearly independent analytic solutions at the origin. To keep the notation 
consistent with that introduced for algebraic functions in [7], we will call this normal behaviour. This normal behaviour follows provided

$$
a_{p}(0) \neq 0
$$

If $a_{p}(0)=0$ then the origin is a singular point and possible solutions will have a different character.

\section{DEFINITION 11 (NORMAL FORM).}

The $\mathbf{n}$ integral form of degree $p, P^{*}(f, x)$, is called normal if

$$
a_{p}(0) \neq 0 \quad\left[\text { or } \partial P^{*}(f, x) /\left.\partial f^{(p)}\right|_{x=0} \neq 0\right] \text {. }
$$

If $p=1$ this condition becomes $a_{1}(0) \neq 0$, which corresponds to the requirement for the D-log approximation in this case. The condition also corresponds to the condition in [2] that the approximation to a meromorphic function be "pole-matching" i.e. that $a_{p}(x)$ vanishes only at the poles of $f$ in $|z|<R$.

Since the solution space of (6) has dimension $p$ it will be necessary to impose $p$ linearly independent conditions in order to distinguish a unique solution, $Q^{*}(x)$, in this space. The usual conditions used to distinguish a unique solution are

$$
Q^{*(i)}(0)=f^{(i)}(0), \quad i=0(1) p-1
$$

We may consider the approximation properties of this unique solution as an approximation to $f(x)$.

\section{THEOREM 12.}

Let $P^{*}(f, x)$ be the normal $\mathbf{n}$ integral form of degree $p$ defined by equation (2), and satisfying $P^{*}(f, x)=O\left(x^{N+S}\right)$ where $S \geq 0$ is the surplus.

Then the unique $\mathbf{n}$ integral function of degree $p, Q^{*}(x)$, defined by the equation

$$
P^{*}\left(Q^{*}, x\right)=0,
$$

subject to the initial conditions

$$
Q^{*(i)}(0)=f^{(i)}(0), \quad i=0(1) p-1,
$$

is an approximation to $f(x)$ satisfying

$$
Q^{*}(x)=f(x)+O\left(x^{N+S+p}\right) .
$$


PROOF:

Let $N+S=R$. Since we have

$$
P^{*}(f, x)=O\left(x^{R}\right), \quad P^{*}\left(Q^{*}, x\right)=0,
$$

then

$$
\left.\frac{d^{i}}{d x^{i}} P^{*}(f, x)\right|_{x=0}=0=\left.\frac{d^{i}}{d x^{i}} P^{*}\left(Q^{*}, x\right)\right|_{x=0}, i=0(1) R-1
$$

For $i=0$ in (8)

$$
\left.P^{*}(f, x)\right|_{x=0}=\left.P^{*}\left(Q^{*}, x\right)\right|_{x=0} .
$$

The initial conditions (7) imply this equation becomes

$$
a_{p}(0) f^{(p)}(0)=a_{p}(0) Q^{*(p)}(0)
$$

and since $P^{*}$ is normal, $a_{p}(0) \neq 0$, and hence

$$
Q^{*(p)}(0)=f^{(p)}(0)
$$

For $i=1$ in (8)

$$
\left.\left[P^{*}\left(f^{(1)}(x), x\right)+\partial P^{*}(f, x) / \partial x\right]\right|_{x=0}=\left.\left[P^{*}\left(Q^{*(1)}(x), x\right)+\partial P^{*}\left(Q^{*}, x\right) / \partial x\right]\right|_{x=0} .
$$

Again using the initial conditions (7) and also (9), this equation reduces to

$$
a_{p}(0) f^{(p+1)}(0)=a_{p}(0) Q^{*(p+1)}(0),
$$

which implies, since $a_{p}(0) \neq 0$,

$$
Q^{*(p+1)}(0)=f^{(p+1)}(0) .
$$

In general,

$$
\left.\left[P^{*}\left(f^{(i)}(x), x\right)+F_{i}\right]\right|_{x=0}=\left.\left[P^{*}\left(Q^{*(i)}(x), x\right)+G_{i}\right]\right|_{x=0}, \quad i=0(1) R-1,
$$

where

$$
\begin{aligned}
& F_{0}=0=G_{0} \\
& F_{1}=\partial P^{*}(f, x) / \partial x=F_{1}\left(x, f(x), f^{(1)}(x), \cdots, f^{(p)}(x)\right) \\
& G_{1}=\partial P^{*}\left(Q^{*}, x\right) / \partial x=F_{1}\left(x, Q^{*}(x), Q^{*(1)}(x), \cdots, Q^{*(p)}(x)\right)
\end{aligned}
$$


and

$$
\begin{aligned}
& F_{i}=\partial P^{*}\left(f^{(i-1)}(x), x\right) / \partial x+d F_{i-1} / d x=F_{i}\left(x, f(x), f^{(1)}(x), \cdots, f^{(p+i-1)}(x)\right) \\
& G_{i}= \\
& \text { for } i=1(1) R-1 .
\end{aligned}
$$

The induction step implies that since

$$
Q^{*(j)}(0)=f^{(j)}(0), \quad j=0(1) p+i-1
$$

then $F_{i}=G_{i}$ and hence, since $P^{*}$ is normal, equation (10) implies that

$$
Q^{*(p+i)}(0)=f^{(p+i)}(0)
$$

It follows that

$$
Q^{*(i)}(0)=f^{(i)}(0), \quad i=0(1) R+p-1
$$

and hence

$$
Q^{*}(x)=f(x)+O\left(x^{R+p}\right)
$$

Hence, in the case that $P^{*}(f, x)$ is a normal form, a unique integral function of degree $p$ is obtained from the unique corresponding integral form of order $R$, and this unique function, $Q^{*}(x)$, approximates $f(x)$ in the neighbourhood of the origin with order of approximation $R+p$.

\section{\$3.1 The Non-Normal Case}

Differential equations in which the corresponding integral form is not normal are not uncommon. A simple example would be Bessel's equation, whose solutions are the well-known Bessel functions. A further example occurs when the integral form has a factor of $x^{r}, r>0$, as was obtained in Theorem 8. This situation leads to an approximation whose order is less than that expected in the normal case.

In [7] a deficiency index for algebraic functions was defined to measure the amount by which the approximation falls short of the expected order. An analogous concept is appropriate in the case of integral functions. 


\section{DEFINITION 13 (DEFICIENCY).}

The deficiency, $D(\mathbf{n})=D \geq 0$ of the $\mathbf{n}$ integral function of degree $p, Q^{*}(x)$, which satisfies $P^{*}(Q, x)=0$, is defined in terms of the corresponding integral form by

$$
\begin{array}{rlr}
\frac{\partial}{\partial f^{(p)}}\left[\frac{d^{k} P^{*}(f, x)}{d x^{k}}\right] & =O\left(x^{D-k}\right) & \text { for } k=0(1) D, \text { as } x \rightarrow 0 \\
\text { and } & \neq O\left(x^{D-k+1}\right) & \text { for at least one value of } k
\end{array}
$$

This condition can also be expressed alternatively in terms of the coefficients of $P^{*}(f, x)$. Thus for $k=0$ we obtain $a_{p}^{*}(x)=O\left(x^{D}\right)$. For $k=1$ we obtain $a_{p-1}^{*}(x)+a_{p}^{*(1)}(x)=$ $O\left(x^{D-1}\right)$, which implies $a_{p-1}^{*}(x)=O\left(x^{D-1}\right)$. For $k=2$,

$$
a_{p-2}^{*}(x)+2 a_{p-1}^{*(1)}(x)+a_{p}^{*(2)}(x)=O\left(x^{D-2}\right)
$$

which may be written as $a_{p-2}^{*}(x)=O\left(x^{D-2}\right)$ using the previous results.

Thus, in general, the conditions for the deficiency, $D$, become

$$
\begin{aligned}
a_{p-k}^{*}(x) & =O\left(x^{D-k}\right) \quad \text { for } k=0(1) D, \quad \text { as } x \rightarrow 0 \\
& \neq O\left(x^{D-k+1}\right) \quad \text { for at least one value of } k .
\end{aligned}
$$

Note that if $D=0$, the integral form is normal. It will be shown that the deficiency, $D$, is the amount by which the order of approximation falls short of the expected order.

Firstly, we consider the case where the integral form has a factor of $x^{r}, r>0$ an integer.

\section{LEMMA 14 (COMMON FACTOR)}

If $P_{\mathbf{n}}^{*}(f, x)=x^{r} P_{\mathbf{m}}^{*}(f, x)$, where $P_{\mathbf{m}}^{*}(f, x)$ has polynomial coefficients with no common factor of $x$ (i.e. $\sum_{i=0}^{p}\left|a_{i}^{*}(0)\right| \neq 0$ in the notation of [7]), then

$$
D=D(\mathbf{n})=D(\mathbf{m})+r
$$

PROOF:

$$
\frac{d^{k} P_{\mathbf{n}}^{*}(f, x)}{d x^{k}}=\sum_{j=0}^{k}\left(\begin{array}{l}
k \\
j
\end{array}\right) \frac{\partial^{j}\left(P_{\mathbf{n}}^{*}\left(f^{(k-j)}, x\right)\right)}{\partial x^{j}}
$$


If the polynomial coefficients of $P_{\mathrm{m}}^{*}(f, x)$ are $a_{i}^{*}(x), i=0(1) p$, then the coefficients of $P_{\mathbf{n}}^{*}(f, x)$ are $x^{r} a_{\mathbf{i}}^{*}(x)$. Applying the operator $\partial / \partial f^{(p)}$ to equation (11), the right side becomes

$$
\sum_{j=0}^{k}\left(\begin{array}{l}
k \\
j
\end{array}\right) \frac{d^{j}}{d x^{j}}\left[x^{r} a_{p-k+j}^{*}(x)\right] .
$$

If $k<r$ then this expression will still have a factor of $x$, and if $k=r$ then the term $k ! a_{p}^{*}(x)$ is the only term without an explicit factor of $x$. Provided $a_{p}^{*}(0) \neq 0$, then by Definition 13 , the integral form $P_{\mathbf{n}}^{*}(f, x)$ has deficiency $r$. However if $a_{p}^{*}(0)=0$, there will be a further factor of $x$. In fact if $a_{p}^{* \prime}(0)=0=a_{p-1}^{*}(0)$ there will be an additional factor of $x$. Continuing in this way, we are simply restating the conditions for the deficiency of $P_{\mathbf{m}}^{*}(f, x)$. That is the total deficiency of $P_{\mathbf{n}}^{*}(f, x)$ willl be $r$ plus the deficiency of $P_{\mathbf{m}}^{*}(f, x)$.

THEOREM 15.

If $P_{\mathbf{n}}^{*}(f, x)=x^{r} P_{\mathbf{m}}^{*}(f, x)$, where $P_{\mathbf{m}}^{*}(f, x)$ is the normal integral form satisfying

$$
P_{\mathbf{m}}^{*}(f, x)=O\left(x^{M+S(\mathbf{m})}\right)
$$

where $S(\mathbf{m})$ is the surplus and $M+1=\sum_{i=0}^{p}\left(m_{i}+1\right)$, then the $\mathbf{n}$ integral function, $Q^{*}(x)$, defined by the equation

$$
P_{\mathbf{n}}^{*}\left(Q^{*}, x\right)=0
$$

subject to the initial conditions

$$
Q^{*(i)}(0)=f^{(i)}(0), \quad i=0(1) p-1
$$

is an approximation to $f(x)$ satisfying

$$
Q^{*}(x)=f(x)+O\left(x^{N+S+p-D}\right)
$$

where $D=D(\mathbf{n}) \geq 0$ is the deficiency of $P_{\mathbf{n}}^{*}(f, x)$,

and $S=S(\mathbf{n}) \geq 0$ is the surplus of $P_{\mathbf{n}}^{*}(f, x)$.

PROOF:

By Lemma $14, D=D(\mathbf{n})=D(\mathbf{m})+r$. Since $P_{\mathbf{m}}^{*}(f, x)$ is normal, $D(\mathbf{m})=0$ and hence $D=r$. 
$P_{\mathbf{n}}^{*}(Q, x)=x^{r} P_{\mathbf{m}}^{*}(Q, x)=0$, and hence the $\mathbf{n}$ integral function of degree $p$ defined by $P_{\mathbf{n}}^{*}(Q, x)=0$ is in fact the same as the $\mathbf{m}$ algebraic function of degree $p$ defined by $P_{\mathbf{m}}^{*}(Q, x)=0$. Hence by Theorem 12 we have

$$
Q^{*}(x)=f(x)+O\left(x^{M+S(\mathbf{m})+p}\right)
$$

By Theorem 8, we have $N=M+(p+1) r$ and

$$
S(\mathbf{n})=S(\mathbf{m})-p r
$$

Hence $M+S(\mathbf{m})+p=N+S(\mathbf{n})+p-r$

As with the case of algebraic functions [7], additional complications can arise beyond the basic definition of the deficiency. As in that previous paper, we illustrate the basic process involved and avoid the additional complications by making the further assumption that in fact each $a_{p-k}^{*}(x)$ does not have further zeros than that needed for the deficiency. That is we assume that

$$
\operatorname{Ord}\left(\frac{\partial P_{\mathbf{n}}^{*}(f, x)}{\partial f^{(p-k)}}\right)=\operatorname{Ord}\left(a_{p-k}^{*}(x)\right)=D-k \text { for } k=0(1) D
$$

Suppose now that $P_{\mathbf{n}}^{*}(f, x)$ is an integral form whose polynomial coefficients have no common factor of $x$ and that $Q(x)$ defined by $P_{\mathbf{n}}^{*}(Q, x)=0$ has deficiency $D>0$. (The subscript $\mathbf{n}$ will be dropped as being understood for the remainder of this section).

\section{THEOREM 16.}

Let $S$ be the surplus of the $\mathbf{n}$ integral form of degree $p, P^{*}(f, x)$, and let $D$ be the deficiency of the corresponding $\mathbf{n}$ integral function defined by $P^{*}(Q, x)=0$.

If $P^{*}(f, x)$ satisfies

(i) $\left.\left[\sum_{i=0}^{p}\left|a_{i}^{*}(x)\right|\right]\right|_{x=0} \neq 0$,

(ii) $\operatorname{Ord}\left(\frac{\partial P^{*}(f, x)}{\partial f^{(p-k)}}\right)=D-k$, for $k=0(1) D$,

then the associated $\mathbf{n}$ integral function of degree $p, Q^{*}(x)$, defined by the equation

$$
P^{*}\left(Q^{*}, x\right)=0
$$

subject to the initial conditions

$$
Q^{*(i)}(0)=f^{(i)}(0), \quad i=0(1)(p-1-D)
$$


and the auxiliary conditions

$$
Q^{*(p+i-D)}(0)=f^{(p+i-D)}(0),
$$

where $i \in\{1,2, \cdots, R-1\}$ satisfies the auxiliary equation

$$
\sum_{j=0}^{D}\left(\begin{array}{l}
i \\
j
\end{array}\right) a_{p-D+j}^{*(j)}(0)=0
$$

is an approximation to $f(x)$ satisfying

$$
Q^{*}(x)=f(x)+O\left(x^{N+S+p-D}\right)
$$

\section{PROOF:}

Firstly note that the auxiliary equation (14) is a polynomial of degree $D$ in the variable $i$, with all the constant coefficients being non-zero on account of condition (ii) on the integral form $P^{*}(f, x)$. We are concerned only with integer solutions in the interval $[1, R-1]$ and it is clear that there can be at most $D$ such solutions, and hence at most $D$ auxiliary conditions (13).

Let $N+S=R$. Following a similar argument to the proof of Theorem 12 we have

$$
P^{*}(f, x)=O\left(x^{R}\right), \quad P^{*}\left(Q^{*}, x\right)=0,
$$

and

$$
\frac{\partial P^{*}(f, x)}{\partial f^{(p-k)}}=O\left(x^{D-k}\right), \quad \frac{\partial P^{*}\left(Q^{*}, x\right)}{\partial Q^{*}(p-k)}=O\left(x^{D-k}\right), \quad k=0(1) D .
$$

From equations (15)

$$
\left.\frac{d^{i}}{d x^{i}} P^{*}(f, x)\right|_{x=0}=0=\left.\frac{d^{i}}{d x^{i}} P^{*}\left(Q^{*}, x\right)\right|_{x=0}, \quad i=0(1) R-1,
$$

and hence

$$
\left.\sum_{j=0}^{i}\left(\begin{array}{l}
i \\
j
\end{array}\right) \frac{\partial^{j} P^{*}\left(f^{(i-j)}(x), x\right)}{\partial x^{j}}\right|_{x=0}=\left.\sum_{j=0}^{i}\left(\begin{array}{c}
i \\
j
\end{array}\right) \frac{\partial^{j} P^{*}\left(Q^{*(i-j)}(x), x\right)}{\partial x^{j}}\right|_{x=0},
$$

From equations (16)

$$
\begin{aligned}
\frac{d^{j}}{d x^{j}}\left(\left.\frac{\partial P^{*}(f, x)}{\left.\partial f^{(p-k)}\right)}\right|_{x=0}=0\right. & =\left.\frac{d^{j}}{d x^{j}}\left(\frac{\partial P^{*}\left(Q^{*}, x\right)}{\partial Q^{*(p-k)}}\right)\right|_{x=0}, \\
k & =0(1) D, j=0(1) D-k-1,
\end{aligned}
$$


and

$$
\left.\frac{d^{D-k}}{d x^{D-k}}\left(\frac{\partial P^{*}(f, x)}{\partial f^{(p-k)}}\right)\right|_{x=0}=\left.\frac{d^{D-k}}{d x^{D-k}}\left(\frac{\partial P^{*}\left(Q^{*}, x\right)}{\partial Q^{*(p-k)}}\right)\right|_{x=0} \neq 0, \quad k=0(1) D .
$$

(This notation is a little redundant, but has been written this way to be consistent with that in [7].)

(a) Consider the case $D=1$.

For $i=0$ in equation (17)

$$
\left.P^{*}(f, x)\right|_{x=0}=\left.P^{*}\left(Q^{*}, x\right)\right|_{x=0} .
$$

Using the initial conditions (12) and equation (18) this reduces to

$$
a_{p-1}^{*}(0)\left(f^{(p-1)}(0)-Q^{*(p-1)}(0)\right)=0
$$

which implies, using (19),

$$
Q^{*(p-1)}(0)=f^{(p-1)}(0)
$$

For $i=1$ in equation (17)

$$
\left.\left[P^{*}\left(f^{(1)}(x), x\right)+\frac{\partial P^{*}(f, x)}{\partial x}\right]\right|_{x=0}=\left.\left[P^{*}\left(Q^{*(1)}(x), x\right)+\frac{\partial P^{*}\left(Q^{*}, x\right)}{\partial x}\right]\right|_{x=0} .
$$

Using the initial conditions (12), the previous result from $i=0$, and equation (18), this reduces to

$$
\left(a_{p-1}^{*}(0)+a_{p}^{*(1)}(0)\right)\left(f^{(p)}(0)-Q^{*(p)}(0)\right)=0
$$

which implies, using (19), or the auxiliary condition (13) if $i=1$ satisfies the auxiliary equation (14),

$$
Q^{*(p)}(0)=f^{(p)}(0)
$$

Proceeding now as in the proof of Theorem 12, we obtain in general from equation (17)

$$
\begin{gathered}
{\left.\left[P^{*}\left(f^{(i)}, x\right)+\left(\begin{array}{c}
i \\
1
\end{array}\right) \frac{\partial P^{*}\left(f^{(i-1)}(x), x\right)}{\partial x}+F_{i}\right]\right|_{x=0}} \\
=\left.\left[P^{*}\left(Q^{*(i)}, x\right)+\left(\begin{array}{l}
i \\
1
\end{array}\right) \frac{\partial P^{*}\left(Q^{*(i-1)}(x), x\right)}{\partial x}+G_{i}\right]\right|_{x=0}, i=2(1) R-1,
\end{gathered}
$$


where

$$
\begin{aligned}
& F_{i}=F_{i}\left(x, f(x), f^{(1)}(x), \cdots f^{(p+i-2)}(x)\right), \\
& G_{i}=F_{i}\left(x, Q^{*}(x), Q^{*(1)}(x), \cdots, Q^{*(p+i-2)}(x)\right) \text { for } i=2(1) R-1 .
\end{aligned}
$$

As before, the term $a_{p}^{*}(x) f^{(p+i)}(x)$ in $P^{*}\left(f^{(i)}(x), x\right)$ vanishes at $x=0$ by (18), and the induction step implies that since

$$
Q^{*(j)}(0)=f^{(j)}(0), \quad j=0(1)(p+i-2),
$$

equation (20) reduces to

$$
\left(a_{p-1}^{*}(0)+i a_{p}^{*(1)}(0)\right)\left(f^{(p+i-1)}(0)-Q^{*(p+i-1)}(0)\right)=0,
$$

which implies, using (19) or the auxiliary condition (13) if this value of $i$ satisfies the auxiliary equation (14),

$$
Q^{*(p+i-1)}(0)=f^{(p+i-1)}(0) \text { for } i=1(1) R-1 \text {. }
$$

It follows that

$$
Q^{*(j)}(0)=f^{(j)}(0), \quad j=0(1) R+p-2
$$

and hence

$$
Q^{*}(x)=f(x)+O\left(x^{R+p-1}\right)=f(x)+O\left(x^{R+p-D}\right)
$$

(b) Let $D=2$.

For $i=0$ in equation (17)

$$
\left.P^{*}(f, x)\right|_{x=0}
$$

Using the initial conditions (12) and equations (18), this reduces to

$$
a_{p-2}^{*}(0)\left(f^{(p-2)}(0)-Q^{*(p-2)}(0)\right)=0,
$$

which implies, using (19),

$$
Q^{*(p-2)}(0)=f^{(p-2)}(0)
$$

For $i=1$ in equation (17), using this result, the initial conditions (12) and equations (18), we obtain

$$
\left(a_{p-2}^{*}(0)+a_{p-1}^{*(1)}(0)\right)\left(f^{(p-1)}(0)-Q^{*(p-1)}(0)\right)=0 .
$$


By (19), $a_{p-2}^{*}(0) \neq 0, a_{p-1}^{*(1)}(0) \neq 0$, and so the first term in this product is non-zero unless $a_{p-2}^{*}(0)+a_{p-1}^{*(1)}(0)=0$. But this latter possibility corresponds to the solution $i=1$ in the auxiliary equation (14). Hence, either from the above equation, or from the auxiliary condition (13), we have

$$
Q^{*(p-1)}(0)=f^{(p-1)}(0)
$$

In general we obtain from equation (17)

$$
\begin{aligned}
{\left[P^{*}\left(f^{(i)}(x), x\right)\right.} & +\left(\begin{array}{l}
i \\
1
\end{array}\right) \frac{\partial P^{*}\left(f^{(i-1)}(x), x\right)}{\partial x} \\
& \left.+\left(\begin{array}{l}
i \\
2
\end{array}\right) \frac{\partial^{2} P^{*}\left(f^{(i-2)}(x), x\right)}{\partial x^{2}}+F_{i}\right]\left.\right|_{x=0}=0
\end{aligned}
$$

and

$$
\begin{aligned}
& {\left[P^{*}\left(Q^{*(i)}(x), x\right)\right.}+\left(\begin{array}{l}
i \\
1
\end{array}\right) \frac{\partial P^{*}\left(Q^{*(i-1)}(x), x\right)}{\partial x} \\
&\left.+\left(\begin{array}{c}
i \\
2
\end{array}\right) \frac{\partial^{2} P^{*}\left(Q^{*(i-2)}(x), x\right)}{\partial x^{2}}+G_{i}\right]\left.\right|_{x=0}=0 \\
& \text { for } i=2(1) R-1,
\end{aligned}
$$

where $F_{i}=F_{i}\left(x, f(x), f^{(1)}(x), \cdots, f^{(p+i-3)}(x)\right)$,

and $\quad G_{i}=F_{i}\left(x, Q^{*}(x), Q^{*(1)}(x), \cdots, Q^{*(p+i-3)}(x)\right)$.

The coefficients of $f^{(p+i)}(x)$ and $f^{(p+i-1)}(x)$ vanish by (18), and hence, since the induction step implies that since

$$
Q^{*(j)}(0)=f^{(j)}(0) \text { for } j=0(1)(p+i-3),
$$

these equations imply

$$
\begin{array}{r}
\left(a_{p-2}^{*}(0)+i a_{p-1}^{*(1)}(0)+\frac{i(i-1)}{2} a_{p}^{*(2)}(0)\right)\left(f^{(p+i-2)}(0)-Q^{*(p+i-2)}(0)\right)=0, \\
\text { for } i=2(1) R-1 .
\end{array}
$$

Either this value of $i$ satisfies the auxiliary equation (14) (in which case we use the auxiliary condition (13)) or else the expression in the first bracket is non-zero. In either case we deduce

$$
Q^{*(p+i-2)}(0)=f^{(p+i-2)}(0) \quad, \quad i=2(1) R-1
$$

It follows that

$$
Q^{*(j)}(0)=f^{(j)}(0), j=0(1) R+p-3
$$


and hence

$$
Q^{*}(x)=f(x)+O\left(x^{R+p-2}\right)=f(x)+O\left(x^{R+p-D}\right) .
$$

(c) The general case follows in a similar fashion.

For $i=0$ in equation (17)

$$
\left.P^{*}(f, x)\right|_{x=0}=\left.P^{*}\left(Q^{*}, x\right)\right|_{x=0} \text {. }
$$

Using the initial conditions (12) and equations (18), this reduces to

$$
a_{p-D}^{*}(0)\left(f^{(p-D)}(0)-Q^{*(p-D)}(0)\right)=0,
$$

which implies, using (19)

$$
Q^{*(p-D)}(0)=f^{(p-D)}(0)
$$

Also from equation (17)

$$
\begin{aligned}
& {\left.\left[\sum_{j=0}^{i}\left(\begin{array}{l}
i \\
j
\end{array}\right) \frac{\partial^{j} P^{*}\left(f^{(i-j)}(x), x\right)}{\partial x^{j}}\right]\right|_{x=0}=\left.\left[\sum_{j=0}^{i}\left(\begin{array}{l}
i \\
j
\end{array}\right) \frac{\partial^{j} P^{*}\left(Q^{*(i-j)}(x), x\right)}{\partial x^{j}}\right]\right|_{x=0}} \\
& \text { for } i=1 \text { (1) } D-1 \text {. }
\end{aligned}
$$

Using the result for $i=0$, the initial conditons (12) and equations (18), this reduces to

$$
\left[\sum_{j=0}^{i}\left(\begin{array}{l}
i \\
j
\end{array}\right) a_{p-D+i}^{*(j)}(0)\right]\left(f^{(p+i-D)}(0)-Q^{*(p+i-D)}(0)\right)=0, \quad i=1(1) D-1 .
$$

If the first term of this product is zero for say $i=k$, then this corresponds to a solution $i=k$ in the auxiliary equation (14). Hence, either because the first term is non-zero, or by using the auxiliary condition for $i=k$, we obtain

$$
Q^{*(p+i-D)}(0)=f^{(p+i-D)}(0), \quad \text { for } i=0(1) D-1 \text {. }
$$

In general, we obtain from equation (17)

$$
\begin{gathered}
{\left.\left[\sum_{j=0}^{i}\left(\begin{array}{l}
i \\
j
\end{array}\right) \frac{\partial^{j} P^{*}\left(f^{(i-j)}(x), x\right)}{\partial x^{j}}+F_{i}\right]\right|_{x=0}} \\
=\left.\left[\sum_{j=0}^{i}\left(\begin{array}{l}
i \\
j
\end{array}\right) \frac{\partial^{j} P^{*}\left(Q^{*(i-j)}(x), x\right)}{\partial x^{j}}+G_{i}\right]\right|_{x=0} \\
\text { for } i=D(1) R-1,
\end{gathered}
$$


where $F_{i}=F_{i}\left(x, f(x), f^{(1)}(x), \cdots, f^{(p+i-D-1)}(x)\right)$,

and $G_{i}=F_{i}\left(x, Q^{*}(x), Q^{*(1)}(x), \cdots, Q^{*(p+i-D-1)}(x)\right)$.

The coefficients of $f^{(p+k)}(x), k=(i-D+1)(1) i$, vanish by (18) and hence the induction step implies that since

$$
Q^{*(j)}(0)=f^{(j)}(0), \quad j=0(1)(p+i-D-1)
$$

these equations imply

$$
\left[\sum_{j=0}^{D}\left(\begin{array}{l}
i \\
j
\end{array}\right) a_{p-D+j}^{*(j)}(0)\right]\left(f^{(p+i-D)}(0)-Q^{*(p+i-D)}(0)\right)=0, \quad i=D(1) R-1 .
$$

Either this value of $i$ satisfies the auxiliary equation (14) (in which case we use the auxiliary condition (13)), or else the expression in the first term of this product is non-zero. In either case we deduce

$$
Q^{*(p+i-D)}(0)=f^{(p+i-D)}(0), \quad i=D(1) R-1
$$

It follows that

$$
Q^{*(j)}(0)=f^{(j)}(0), \quad j=0(1)(R-p-D-1)
$$

and hence

$$
Q^{*}(x)=f(x)+O\left(x^{R+p-D}\right)
$$

\section{COROLLARY 17.}

For an integral form satisfying the conditions of Theorem 16 , the deficiency, $D$, satisfies $D \leq p$, and the order of approximation by an integral function will always be at least $R$. That is, at worst, $Q^{*}(x)$ satisfies

$$
Q^{*}(x)=f(x)+O\left(x^{R}\right) .
$$




\section{PROOF:} include

The integral form $P^{*}(f, x)$ satisfies $\left.\left[\sum_{i=0}^{p}\left|a_{i}(x)\right|\right]\right|_{x=0} \neq 0$. The hypotheses of the theorem

$$
\operatorname{Ord}\left(\frac{\partial P^{*}(f, x)}{\partial f^{(p-k)}}\right)=D-k, \quad k=0(1) D \text {. }
$$

For $D=p$ this means that

$$
\operatorname{Ord}\left(\frac{\partial P^{*}(f, x)}{\partial f}\right)=0 \text { for } k=D .
$$

That is $a_{0}(x)=O(1)$ and $a_{0}(x) \neq O(x)$. Thus $a_{0}(0) \neq 0$.

If $D>p$ then $a_{0}(x)$ would have a factor of $x$, which contradicts the condition above that not all coefficients $a_{k}(x)$ vanish at the origin. If there were a common factor of $x^{r}, r>0$, for all coefficients, then Theorem 15 would apply.

The techniques used in the above proofs have been modelled after the approach in [7] for algebraic functions. However, viewed as a method for the solution of differential equations, the approach used is similar to the method of Frobenius [6] for the series solution of linear homogeneous differential equations. Thus the integral form is normal if the origin is an ordinary point of the associated integral function. The deficiency of the solution corresponds to the origin being a regular singular point. However it is relevant to note that we are not concerned with finding all solutions of the differential equation, but only with choosing from the solution space that solution which approximates the given locally analytic function. Hence we seek only analytic solutions even though other solutions may have singularities at the origin. The auxiliary equation (14) is clearly closely related to the indicial equation [6].

There may be other singular points away from the origin as was the case for algebraic functions. For quadratic algebraic functions, a preliminary analysis of the behaviour at such points was given in $[3,4]$ and more details will be given in forthcoming reports.

The fundamental process for the order of approximation of a non-normal integral function may be summarized by the following theorem.

\section{THEOREM 18.}

Let $S$ be the surplus and $D$ be the deficiency of the $\mathbf{n}$ integral form of degree $p, P_{\mathbf{n}}^{*}(f, x)$.

Let $P_{\mathbf{n}}^{*}(f, x)=x^{r} P_{\mathbf{m}}^{*}(f, x), r$ a non-negative integer, where $P_{\mathbf{m}}^{*}(f, x)$ has polynomial coefficients $a_{i}^{*}(x)$ which satisfy

(i) $\left.\left[\sum_{i=0}^{p}\left|a_{i}^{*}(x)\right|\right]\right|_{x=0} \neq 0$, 
(ii) $\operatorname{Ord}\left(\frac{\partial P_{\mathbf{m}}^{*}(f, x)}{\partial f^{(p-k)}}\right)=D_{1}-k$ for $k=0(1) D_{1}$, where $D(\mathbf{m})=D_{1}$.

Then the associated $\mathbf{n}$ integral function of degree $p, Q^{*}(x)$, defined by the equation

$$
P_{\mathbf{n}}^{*}\left(Q^{*}, x\right)=0
$$

subject to the initial conditions

$$
Q^{*(i)}(0)=f^{(i)}(0), \quad i=0(1)\left(p-1-D_{1}\right)
$$

and the auxiliary conditions

$$
Q^{*\left(p+i-D_{1}\right)}(0)=f^{\left(p+i-D_{1}\right)}(0)
$$

where $i \in\{1,2, \cdots, R-1\}$ satisfies the auxiliary equation

$$
\sum_{j=0}^{D_{1}}\left(\begin{array}{l}
i \\
j
\end{array}\right) a_{p-D_{1}+j}^{*(j)}(0)=0
$$

is an approximation to $f(x)$ satisfying

$$
Q^{*}(x)=f(x)+O\left(x^{N+S+p-D}\right)
$$

\section{PROOF:}

By Lemma $14, D=D(\mathbf{m})+r=D_{1}+r$. By combining the results of Theorems 15 and 16 the result is obtained.

This section has developed theorems for the basic behaviour of the order of approximation by an $\mathbf{n}$ integral function of degree $p$, determined by collocation at the single node $x=0$. A simple change of variable to $x-x_{0}$ will generalize these results to collocation at an arbitrary node $x_{0}$. It is interesting to observe the close analogy of these results with the case of approximation by algebraic functions [7]. For algebraic functions it was found that the order of approximation was determined by the expression $N+S-D$, while for the case of integral functions of degree $p$ it has been shown that the order of approximation is determined by the expression $N+S+p-D$. It is conjectured that these basic results will also hold true for the cases of more general deficiencies than the basic case that was considered in this report. These details will be considered in a future report. 


\section{\$4. Examples.}

This section contains some illustrative examples of the results of the previous sections. The first two examples consider the case of first order equations, $p=1$.

\section{EXAMPLE 19.}

This is the same as Example 10 in Section 2.

(a) The $(1,0)$ integral form for $f(x)=\cos x$ is

$$
x f(x)+f^{(1)}(x)=O\left(x^{3}\right),
$$

with $p=1, n_{0}=1, n_{1}=0, N=2$ and $S=1$. This form is normal since $\partial P^{*}(f, x) /\left.\partial f^{(1)}\right|_{x=0}=1 \neq 0$.

The corresponding $(1,0)$ integral function $Q^{*}(x)$ is defined by

$$
x Q^{*}(x)+Q^{*(1)}(x)=0,
$$

subject to the initial condition

$$
Q^{*}(0)=f(0)=\cos 0=1 \text {. }
$$

The solution to this initial value problem is

$$
\begin{aligned}
Q^{*}(x)=e^{-x^{2} / 2} & =1-\frac{x^{2}}{2}+\frac{x^{4}}{8} \cdots \\
& =\cos x+O\left(x^{4}\right) \\
& =f(x)+O\left(x^{N+S+p}\right),
\end{aligned}
$$

which illustrates Theorem 12 .

(b) The $(2,1)$ integral form of degree $p=1$ is

$$
x^{2} f(x)+x f^{(1)}(x)=O\left(x^{4}\right),
$$

with $N=4, S=0$. In this case $\partial P^{*}(f, x) / \partial f^{(1)}=x$ and hence $D=1$. However Lemma 14 applies and hence $D=1=r$. Thus applying Theorem 15 and using part (a), we have

$$
Q^{*}(x)=e^{-x^{2} / 2}=\cos x+O\left(x^{4}\right)=f(x)+O\left(x^{N+S+p-D}\right) .
$$


EXAMPLE 20.

Let $p=1, f(x)=(1+x)^{-1 / 3}$.

(a) The $(0,0)$ integral form is

$$
f(x)+3 f^{(1)}(x)=O\left(x^{1}\right)
$$

with $n_{0}=n_{1}=0, N=1$, and $S=0$. This form is normal with $D=0$. The $(0,0)$ integral function satisfies

$$
Q^{*}(x)+3 Q^{*(1)}(x)=0
$$

subject to the initial condition

$$
Q^{*}(0)=f(0)=1
$$

Thus

$$
\begin{aligned}
Q^{*}(x)=e^{-x / 3} & =1-\frac{x}{3}+\frac{x^{2}}{18}+\cdots \\
& =f(x)+O\left(x^{2}\right) \\
& =f(x)+O\left(x^{N+S+p}\right)
\end{aligned}
$$

as in Theorem 12.

(b) The $(0,1)$ integral form is

$$
f(x)+3(1+x) f^{(1)}(x)=0
$$

which implies that $f(x)$ is an integral function of this type as expected. That is, these approximations reproduce integral functions as noted in the text.

The next examples consider how these results extend to the case of quadratic integral function approximation, i.e. $p=2$ with the approximation being the solution of a linear homogeneous differential equation of the second order.

\section{EXAMPLE 21.}

Let $f(x)=\exp (\exp (x))$ and $p=2$. The $(1,1,1)$ integral form for this function is

$$
(x+3) f(x)+3(x+1) f^{(1)}(x)+(x-3) f^{(2)}(x)=O\left(x^{5}\right) \text {. }
$$

For this example $N=5$ and $S=0$. 
Since $\partial P^{*}(f, x) /\left.\partial f^{(2)}\right|_{x=0}=\left.a_{2}^{*}(x)\right|_{x=0}=\left.(x-3)\right|_{x=0}=-3 \neq 0$, the integral form is normal and $D=0$.

The $(1,1,1)$ integral function satisfies

$$
(x+3) Q^{*}(x)+3(x+1) Q^{*(1)}(x)+(x-3) Q^{*(2)}(x)=0,
$$

subject to the initial conditions

$$
\begin{aligned}
& Q^{*}(0)=f(0)=e, \\
& Q^{*(1)}(0)=f^{(1)}(0)=e .
\end{aligned}
$$

We obtain

$$
\begin{aligned}
Q^{*}(x) & =e\left[1+x+2 \frac{x^{2}}{2 !}+5 \frac{x^{3}}{3 !}+15 \frac{x^{4}}{4 !}+52 \frac{x^{5}}{5 !}+203 \frac{x^{6}}{6 !}+\frac{2635}{3} \frac{x^{7}}{7 !}+\cdots\right] \\
& =f(x)+O\left(x^{7}\right) \\
& =f(x)+O\left(x^{N+S+p}\right)
\end{aligned}
$$

in agreement with Theorem 12.

EXAMPLE 22.

Let $f(x)=\log \cos x$ and $p=2$. The $(2,2,2)$ integral form is

$$
\left(32 x^{2}+456\right) f(x)-261 x f^{(1)}(x)+33 x^{2} f^{(2)}(x)=O\left(x^{8}\right)
$$

with $n_{0}=n_{1}=n_{2}=2$ and hence $N=8$ and $S=0$. This integral form is not normal since $\partial P^{*}(f, x) /\left.\partial f^{(2)}\right|_{x=0}=0$. The coefficient polynomials do not have a common factor of $x$ and since

$$
\begin{aligned}
& a_{2}^{*}(0)=0, a_{2}^{*(1)}(0)=0, a_{2}^{*(2)}(0)=66 \\
& a_{1}^{*}(0)=0, a_{1}^{*(1)}(0)=-261, \\
& a_{0}^{*}(0)=456
\end{aligned}
$$

the hypotheses of Theorem 16 are satisfied with $D=2$. The auxiliary equation (14) is thus

$$
456-261 i+33 i(i-1)=0
$$

which has roots $2,76 / 11$. The only integer solution in the range $[1,7]$ is $i=2$. Thus we have one auxiliary condition (13) for $i=2$, and since $p=D=2$ we have no initial conditions in this case.

The $(2,2,2)$ integral function satisfies

$$
\left(32 x^{2}+456\right) Q^{*}(x)-261 x Q^{*(1)}(x)+33 x^{2} Q^{*(2)}(x)=0
$$


subject to the auxiliary condition

$$
Q^{*(2)}(0)=f^{(2)}(0)=-1
$$

Hence we obtain the series solution

$$
\begin{aligned}
Q^{*}(x) & =-\frac{x^{2}}{2}-\frac{x^{4}}{12}-\frac{x^{6}}{45}+\frac{4 x^{8}}{1215}+\cdots \\
& =f(x)+O\left(x^{8}\right) \\
& =f(x)+O\left(x^{N+S+p-D}\right)
\end{aligned}
$$

which illustrates Theorem 16.

EXAMPLE 23.

Let $f(x)=\sum_{n=0}^{5} x^{n} /(n !(n+1) !)+x^{6} \exp (\exp (x))$ with $p=2$.

Let $n_{0}=1=n_{1}, n_{2}=2$ and hence $N=6$. The $(1,1,2)$ integral form is

$$
-x f(x)+2 x f^{(1)}(x)+x^{2} f^{(2)}(x)=O\left(x^{6}\right)
$$

with $S=0$.

This integral form is not normal since $\partial P^{*}(f, x) /\left.\partial f^{(2)}\right|_{x=0}=0$. According to Lemma 14, we may take out a common factor of $x$, so $r=1$. The resulting $(0,0,1)$ integral form is

$$
-f(x)+2 f^{(1)}(x)+x f^{(2)}(x)=O\left(x^{5}\right)
$$

(with $S(\mathbf{m})=2$ ), and this is still not normal since $\partial P_{\mathbf{m}}^{*}(f, x) / \partial f^{(2)}=x$. However $a_{2}^{*}(0)=$ $0, a_{2}^{*(1)}(0)=1, a_{1}^{*}(0)=2$, and so Theorem 18 applies with $D_{1}=1$.

The auxiliary equation (14) is thus

$$
2+i 1=0
$$

whose only solution, $i=-2$, does not lie in $[1,5]$ and hence auxiliary conditions are not applicable.

Hence the $(1,1,2)$ integral function satisfies

$$
-Q^{*}(x)+2 Q^{*(1)}(x)+x Q^{*(2)}(x)=0,
$$

subject to the initial condition

$$
Q^{*}(0)=f(0)=1
$$


The solution in this case is

$$
\begin{aligned}
Q^{*}(x) & =\sum_{n=0}^{5} x^{n} /(n !(n+1) !)+x^{6} /(6 ! 7 !)+\cdots \\
& =f(x)+O\left(x^{6}\right) \\
& =f(x)+O\left(x^{N+S+p-D}\right)
\end{aligned}
$$

in accordance with Theorem 18 , since $D=D_{1}+r=2$.

This section of examples is concluded with an example of an integral function of higher degree with $p=4$.

\section{EXAMPLE 24.}

Let $f(x)=\sum_{n=0}^{8} x^{n} /(n !(n+1) !)+x^{9} \exp (x) / 9 !$ with $p=4$.

Let $n_{0}=n_{1}=n_{2}=0, n_{3}=n_{4}=2$ and hence $N=8$. The $(0,0,0,2,2)$ integral form is

$$
P^{*}(f, x) \equiv-f(x)+9 f^{(1)}(x)-21 f^{(2)}(x)-3 x f^{(3)}(x)+x^{2} f^{(4)}(x)=O\left(x^{8}\right)
$$

with $S=0$.

This integral form is not normal since

$$
\partial P^{*}(f, x) /\left.\partial f^{(4)}\right|_{x=0}=\left.x^{2}\right|_{x=0}=0
$$

The coefficient polynomials do not have a common factor of $x$, but satisfy

$$
\begin{aligned}
& a_{4}^{*}(0)=0, \quad a_{4}^{*(1)}(0)=0, \quad a_{4}^{*(2)}(0)=2, \\
& a_{3}^{*}(0)=0, \quad a_{3}^{*(1)}(0)=-3, \\
& a_{2}^{*}(0)=-21 .
\end{aligned}
$$

Hence the hypotheses of Theorem 16 are satisfied with $D=2$. The auxiliary equation (14) is thus

$$
-21-3 i+i(i-1)=0
$$

whixh has roots $i=-3,7$. The only integer solution in the range $[1,7]$ is $i=7$. Thus we have one auxiliary condition (13) for $i=7$. Since $p=4, D=2, i=7, p+i-D=9$, the auxiliary condition is for the 9 th derivative.

The integral function approximation is defined by the equation

$$
P^{*}\left(Q^{*}, x\right)=0
$$


subject to the initial conditions

$$
\begin{aligned}
& Q^{*}(0)=f(0)=1, \\
& Q^{*(1)}(0)=f^{(1)}(0)=\frac{1}{2},
\end{aligned}
$$

and the auxiliary condition

$$
Q^{*(9)}(0)=f^{(9)}(0)=1
$$

The series solution of this differential equation is

$$
\begin{aligned}
Q^{*}(x) & =\sum_{n=0}^{8} x^{n} /(n !(n+1) !)+x^{9} / 9 !-x^{10}(3265919 / 11 ! 9 !)+\cdots \\
& =f(x)+O\left(x^{10}\right) \\
& =f(x)+O\left(x^{N+S+p-D}\right)
\end{aligned}
$$

as given by Theorem 16 .

\section{\$5. Conclusion.}

This paper has considered the problem of approximating a real-valued, locally analytic function, $f(x)$, by an integral function $Q(x)$. Following the analogous procedure in [7], a careful distinction was made between the approximating properties of the integral form, by which the polynomial coefficients of the integral function are defined, and the approximating properties of the integral function itself.

By defining a surplus $S$ for the integral form a unique integral form could be defined. It was shown how a basic structure within a table of integral forms could be constructed, but the global nature of such a table is complicated by the possibility of overlapping structures.

A concept of the deficiency $D$ of the integral function was also defined. This is analogous to the concept of deficiency introduced in [7] for algebraic functions. A normal integral function (which corresponds to the differential equation having the origin as an ordinary point) has deficiency $D=0$, and there is unique element in the solution space with specified approximating properties. For a non-normal integral function of degree $p$ (with $D \neq 0$ ) it was shown that a unique element in the solution space could still be determined with the order of approximation determined by the expression $N+S+p-D$. This may be compared to the case of algebraic functions where it has been shown [7] that the order of approximation is determined by the expression $N+S-D$. 
Although these results have been obtained for a function locally analytic at the origin, it is clear that a simple change of variable to $x-x_{0}$ will generalize the results for a function which is locally analytic at an arbitrary point $x_{0}$.

Just as the algebraic form reproduced algebraic functions, as might be reasonably expected, so the integral form reproduces integral functions. This was illustrated by the example of an algebraic function which was reproduced as an integral function.

The results obtained have been illustrated by some simple examples in Section 4 .

\section{Acknowledgement}

The author wishes to acknowledge the hospitality of the Center for Applied Mathematics, Purdue University, where this research was initiated.

\section{References}

1. Baker, G.A., Graves-Morris, P.R. : Padé Approximants, Part I: Basic Theory, Part II: Extensions and Applications. Reading, MA: Addison-Wesley 1981

2. Baker, G.A., Lubinsky, D.S. : Convergence theorems for rows of differential and algebraic Hermite-Padé approximations. J. Comput. Appl. Math. 18, 29-52 (1987)

3. Brookes, R.G., McInnes, A.W. : Some qualitative results for the quadratic function approximation. Research Report 46, Department of Mathematics, University of Canterbury (1988)

4. Brookes, R.G., McInnes, A.W. : More qualitative results for the quadratic function approximation. Research Report 47, Department of Mathematics, University of Canterbury (1989)

5. Della Dora, J,, Di Crescenzo, C. : Approximations de Padé-Hermite. Numer. Math. 43, 23-29 and 41-57 (1984)

6. Ince, E.L. : Ordinary Differential Equations. New York : Dover 1956

7. McInnes, A.W. : Existence and uniqueness of algebraic function approximations. Research Report 50, Department of Mathematics, University of Canterbury (1989) 
8. McInnes, A.W. : The existence of approximations by algebraic functions determined by collocation. Technical Report 112, Center for Applied Mathematics, Purdue University (1989)

9. Stahl, H. : Asymptotics of Hermite-Padé polynomials and related convergence results - a summary of results. In: Cuyt, A. (ed.) Nonlinear Numerical Methods and Rational Approximation, pp.25-53. Dordrecht, Netherlands: D. Reidel Publishing Company 1988 